\title{
Lie group analysis and similarity solutions for hydro-magnetic Maxwell fluid through a porous medium
}

\author{
Khaled Saad Mekheimer ${ }^{1 *}$, Mostafa Fatouh El-Sabbagh ${ }^{2}$ and Rabea Elshennawy Abo-Elkhair ${ }^{1}$
}

\author{
* Correspondence: \\ kh_Mekheimer@yahoo.com \\ ${ }^{1}$ Mathematics Department, Faculty \\ of Science, Al-Azhar University, \\ Nasr City 11448, Cairo, Egypt \\ Full list of author information is \\ available at the end of the article
}

\begin{abstract}
The equations of two dimensional incompressible fluid flow for hydro-magnetic Maxwell fluid through a porous medium have been studied. Lie group analysis has been employed and the group invariant solutions are obtained. Solutions corresponding to translational and rotational symmetries are obtained. A boundary value problem for the translational symmetry is investigated and the results are also sketched graphically. The effects of physical parameters have been noticed. MSC 2011: 53C11; 76S05.

Keywords: lie point symmetries, similarity solutions, Maxwell fluid, porous medium, $\mathrm{MHD}$
\end{abstract}

\section{Introduction}

Non-Newtonian fluid behavior, which is characterized by a nonlinear viscosity dependence on the strain, can be observed in many complex fluids, for example, polymers, dense colloidal dispersions, surfactant solutions, micellar solutions chemical, and petroleum industries [1]. In addition to shear-thinning and shear-thickening behavior, a dynamic or even chaotic response can be found in some fluids subjected to a steady shear flow. Because of the difficulty to suggest a single model which exhibits all properties of non-Newtonian fluids, they cannot be described as simply as Newtonian fluids. Due to this fact many models of constitutive equations have been proposed and most of them are empirical or semi empirical [2]. Amongst these the differential type fluid model gained considerable attention of many researchers. The flows of non-Newtonian fluids are not only important because of their technological significance but also in the interesting mathematical features presented by the equations governing the flow. However on the other hand there are much controversies on these models as well. Such fluids are also inadequate to describe the relaxation phenomena. For a complete and detailed discussion of the relevant issues for differential type fluids, we refer the readers to Dunn and Rajagopal [3] and Aksel [4].

The non-Newtonian fluids are mainly classified into three types namely differential, rate and integral. The simplest subclass of the rate type fluids is the Maxwell model [5]. This fluid model can very well describe the relaxation time effects. Specifically the Maxwell fluid model has been used for the viscoelastic flows where the dimensionless

(C) 2012 Mekheimer et al; licensee Springer. This is an Open Access article distributed under the terms of the Creative Commons Attribution License (http://creativecommons.org/licenses/by/2.0), which permits unrestricted use, distribution, and reproduction in any medium, provided the original work is properly cited. 
relaxation time is small. However in some more concentrated polymeric fluids the Maxwell model is also useful for large dimensionless relaxation time. Some recent investigations dealing with the flows of Maxwell fluids are given in the references [6-9].

Modified Darcy's law for a Maxwell fluid including the Hall current has been used for the modeling. In fact, the Hall effect is important when the Hall parameter, which is the ratio between the electron-cyclotron frequency and the electronatom-collision frequency, is high. This happens, when the magnetic field is high or when the collision frequency is low. In most cases, the Hall term has been ignored in applying Ohm's law as it has no marked effects for small and moderate values of the magnetic field. However, the current trend in the application of magnetohydrodynamics is towards a strong magnetic field, so that the influence of electromagnetic force is noticeable. Under these conditions, the Hall current is important and it has marked effects on the magnitude and direction of the current density and consequently on the magnetic-force term. Therefore, it is of interest to study the influence of the Hall current on the flow.

In the Earth there are a large number of problems that can be described by the interaction of a low viscosity fluid (water, oil, gas, magma) in a permeable (possibly deformable) matrix. Darcy's Law is the classic, empirically derived equation for the flux of a low viscosity fluid in a permeable matrix. This equation assumes that flow in the pores or cracks of the medium is essentially laminar and provides the average flux through a representative area that is larger than the pore scale and smaller than the scale of significant permeability variation (if such a scale exists). Various approaches have been used to justify this rule from first principles (see e.g., Dagan [10]) but it generally seems to work.

In this article, we apply the so-called symmetry methods for a particular problem of fluid mechanics. The main advantage of such methods is that they can successfully be applied to nonlinear differential equations [11-13]. The similarity solutions are quite popular because they result in the reduction of the independent variables of the problem. The symmetry transformations method transform the given family of equations of $n$ independent variables, say, to another family of equations of $n-1$ independent variables, which can further be solved $[14,15]$. The fundamental concepts of this approach can be found in [16-19]. In our case, the problem under investigation is $(2+$ 1)-nonlinear partial differential equations (PDEs). Hence, any similarity solution will transform the system of $(n+1)$-nonlinear PDEs into a system of $(n)$-nonlinear PDEs and any similarity solution will transform the system of (2)-nonlinear PDEs into a system of ordinary differential equations (ODEs).

Many authors used Lie group analysis method to obtain the exact solutions for some problems in fluid mechanics. Yurusoy and Pakdemirli [20] investigated the boundary layer equations of a non-Newtonian fluid model in which the shear stress is an arbitrary function of the velocity gradient. Yurusoy et al. [21] have obtained the solution for the creeping flow of the second grade fluid. Also the two-dimensional equations of motions for the slowly flowing and heat transfer in second grade fluid in cartesian coordinates neglecting the inertial terms are considered by Yürüsoy [22]. Shahzad et al. [23] found the analytical solution of a micropolar fluid by using the Lie group analysis. Recently, Mekheimer et al. studied the Lie group analysis and similarity solutions for a couple stress fluid with heat transfer [24], Lie point symmetries and similarity solutions for an electrically conducting Jeffrey fluid [25] and Lie point symmetries and similarity solution for a micro-polar fluid through a porous medium [26]. 
From discussion above, we attend to find the analytical (similarity) solutions for the flow problem of an incompressible hydro-magnetic Maxwell fluid through a porous medium using Lie group analysis. The problem is presented as follows, in Section 2, the equations governing two-dimensional motion of an incompressible, MHD Maxwell fluid are introduced. In Section 3, the basic idea of the Lie group analysis method are given and used to find the isovector field of our equations. The similarity solutions corresponding to translational and rotational symmetry are obtained in Sections 3.1 and 3.2. Also a boundary value problem for the similarity solutions corresponding to translational symmetry are obtained in Section 4 . The graphs for a boundary value problem (magma flow) are plotted and discussed in Section 5. Finally a concluding remarks are pointed in Section 6.

\section{Equations of motion}

The continuity and momentum equations governing the two-dimensional motion of an incompressible hydro-magnetic Maxwell fluid through a porous medium can be written as:

$$
\begin{aligned}
E_{1}= & \frac{\partial \tilde{u}}{\partial \tilde{x}}+\frac{\partial \tilde{v}}{\partial \tilde{y}}=0, \\
E_{2}= & \left(1+\tilde{\lambda} \frac{\partial}{\partial t}\right)\left[\frac{\partial \tilde{u}}{\partial \tilde{t}}+\tilde{u} \frac{\partial \tilde{u}}{\partial \tilde{x}}+\tilde{v} \frac{\partial \tilde{u}}{\partial \tilde{y}}\right]+\left(1+\tilde{\lambda} \frac{\partial}{\partial \tilde{t}}\right) \frac{1}{\rho} \frac{\partial \tilde{p}}{\partial \tilde{x}}-\frac{\mu}{\rho} \nabla^{2} \tilde{u} \\
& +\left(1+\tilde{\lambda} \frac{\partial}{\partial \tilde{t}}\right)\left[\frac{\sigma B_{0}^{2} \theta}{\rho}(\tilde{u}-m \tilde{v})\right]+\frac{\mu \varphi}{\rho \tilde{k}} \tilde{u}=0, \\
E_{3}= & \left(1+\tilde{\lambda} \frac{\partial}{\partial \tilde{t}}\right)\left[\frac{\partial \tilde{v}}{\partial \tilde{t}}+\tilde{u} \frac{\partial \tilde{v}}{\partial \tilde{x}}+\tilde{v} \frac{\partial \tilde{v}}{\partial \tilde{y}}\right]+\left(1+\tilde{\lambda} \frac{\partial}{\partial \tilde{t}}\right) \frac{1}{\rho} \frac{\partial \tilde{p}}{\partial \tilde{y}}-\frac{\mu}{\rho} \nabla^{2} \tilde{v} \\
& +\left(1+\tilde{\lambda} \frac{\partial}{\partial \tilde{t}}\right)\left[\frac{\partial B_{0}^{2} \theta}{\rho}(\tilde{v}+m \tilde{u})\right]+\frac{\mu \varphi}{\rho \tilde{v}} \tilde{v}=0,
\end{aligned}
$$

where $\nabla^{2}=\frac{\partial^{2}}{\partial \tilde{x}^{2}}+\frac{\partial^{2}}{\partial \tilde{y}^{2}}, \theta=\frac{1}{1+m^{2}}, m=\frac{\partial B_{0}}{e n_{e}}$ are the fluid velocities in the $\tilde{x}, \tilde{y}$ directions, $\tilde{p}$ is the pressure, and $\tilde{t}$ is the time. Here $\tilde{\lambda}, \rho, \mu, \phi, k, e, n_{e}, \sigma, B_{0}$, and $m$ are the relaxation time, density, coefficient of viscosity, porosity of the porous medium, permeability, electric charge, the number density of electrons, electrical conductivity of the fluid, magnetic field and Hall parameter respectively.

Using the following dimensionless parameters

$$
u=\frac{\tilde{u}}{U^{\prime}} \quad v=\frac{\tilde{v}}{U^{\prime}}, \quad x=\frac{\tilde{x}}{L}, \quad y=\frac{\tilde{y}}{L}, \quad t=\frac{U}{L} \tilde{t}, \quad p=\frac{\tilde{p}}{\rho U^{2}}, \quad \lambda=\frac{U}{L} \tilde{\lambda}, \quad k=\frac{\tilde{k}}{\varphi L^{2}},
$$

the system (1) becomes

$$
\begin{aligned}
E_{1}= & \frac{\partial u}{\partial x}+\frac{\partial v}{\partial y}=0 \\
E_{2}= & \left(1+\lambda \frac{\partial}{\partial t}\right)\left[\frac{\partial u}{\partial t}+u \frac{\partial u}{\partial x}+v \frac{\partial u}{\partial y}\right]+\left(1+\lambda \frac{\partial}{\partial t}\right) \frac{\partial p}{\partial x}-\frac{1}{R} \nabla^{2} u \\
& +\left(1+\lambda \frac{\partial}{\partial t}\right)[M \theta(u-m v)]+\frac{1}{R k} u=0, \\
E_{3}= & \left(1+\lambda \frac{\partial}{\partial t}\right)\left[\frac{\partial v}{\partial t}+u \frac{\partial v}{\partial x}+v \frac{\partial v}{\partial y}\right]+\left(1+\lambda \frac{\partial}{\partial t}\right) \frac{\partial p}{\partial y}-\frac{1}{R} \nabla^{2} v \\
& +\left(1+\lambda \frac{\partial}{\partial t}\right)[M \theta(v+m u)]+\frac{1}{R k} v=0,
\end{aligned}
$$


where $R=\frac{\rho L U}{\mu}$ is the Reynolds number, $M=\frac{\sigma B_{0}^{2} L}{\rho U}$ is the Hartmann number and $L$, $U$ are the dimensionless length and velocity, respectively.

\section{Lie group analysis and isovector fields}

In order to obtain the analytical solution, we apply the Lie group analysis theory to system (3). For this we write

$$
\left\{\begin{array}{c}
x_{i}^{*}=x_{i}+\varepsilon \xi_{i}\left(x_{j}, u_{\alpha}\right)+\mathrm{o}\left(\varepsilon^{2}\right), \\
u_{\alpha}^{*}=u_{\alpha}+\varepsilon \eta_{\alpha}\left(x_{j}, u_{\beta}\right)+\mathrm{o}\left(\varepsilon^{2}\right),
\end{array} \quad i, j=1,2,3, \quad \alpha, \beta=1,2,3,\right.
$$

as the infinitesimal Lie point transformations. We have assumed that system (3) is invariant under the transformations given in Eq. (4). The corresponding infinitesimal generator of Lie groups (symmetries) is given by

$$
X=\xi_{i} \frac{\partial}{\partial x_{i}}+\eta_{\alpha} \frac{\partial}{\partial u_{\alpha}}
$$

with summation convention over the repeated index and $x_{1}=x, x_{2}=y, x_{3}=t, u_{1}=$ $u, u_{2}=v, u_{3}=p$. The coefficients $\xi_{1}, \xi_{2}, \xi_{3}, \eta_{1}, \eta_{2}$, and $\eta_{3}$ are the functions of all independent and dependent variables. There coefficients are the components of the infinitesimals symmetries corresponding to $x, y, t, u, v$, and $p$, respectively to be determined from the invariance conditions:

$$
\left.\operatorname{Pr}^{(2)} X\left(E_{a}\right)\right|_{E_{\alpha=0}}=0, \quad a=1,2,3,
$$

where $E_{a}=0, i=1,2,3$ represent the system of Eq. (3) and $\operatorname{Pr}^{(2)}$ is the second prolongation of the isovector field $X$. Since the system (3) is of order two, then our prolongation will be in the form

$$
\begin{aligned}
& \operatorname{Pr}^{(1)} X=X+\eta_{\alpha i} \frac{\partial}{\partial u_{\alpha, i}} \\
& \operatorname{Pr}^{(2)} X=\operatorname{Pr}^{(1)} X+\eta_{\alpha i j} \frac{\partial}{\partial u_{\alpha, i j}} .
\end{aligned}
$$

where

$$
\begin{aligned}
& \eta_{\alpha i}=D_{i}\left[\eta_{\alpha}-\xi_{j} u_{\alpha, j}\right]+\xi_{j} u_{\alpha, j i} \\
& \eta_{\alpha i j}=D_{i j}\left[\eta_{\alpha}-\xi_{k} u_{\alpha, k}\right]+\xi_{k} u_{\alpha, k i j .}
\end{aligned}
$$

and the operator $D_{i_{1} i_{2 . . i}}$ is called the total derivative (Hash operator) and has the following form:

$$
D_{i}=\frac{\partial}{\partial x_{i}}+u_{\alpha, i} \frac{\partial}{\partial u_{\alpha}}+u_{\alpha, i j} \frac{\partial}{\partial u_{\alpha, j}}+u_{\alpha, i j k} \frac{\partial}{\partial u_{\alpha, j k}},
$$

where $D_{i j}=D_{i}\left(D_{j}\right)=D_{j}\left(D_{i}\right)=D_{j i}$ and $u_{\alpha, i}=\frac{\partial u_{\alpha}}{\partial x_{i}}$.

Expanding the system of Eq. (6) with the aid of Mathematica programm, along with the original system of Eq. (3) to eliminate $u_{x}, p_{x t} p_{y t}$ and setting the coefficients involving $u_{y}, u_{y y}, v_{x}, v_{y}, v_{x x}, v_{x y}, v_{y y}$ and various products to zero give rise the essential set of 
over-determined equations. Solving these set of determining equations we obtain the required components of isovector field as follows:

$$
\begin{aligned}
& \xi_{1}=a_{2}-a_{1} y, \quad \xi_{2}=a_{3}+a_{1} x, \quad \xi_{3}=a_{4}, \\
& \eta_{1}=-\alpha_{1} v, \quad \eta_{2}=a_{1} u, \quad \eta_{3}=\delta(t),
\end{aligned}
$$

where $a_{i}, i=1, \ldots, 5$ are arbitrary constants, $\delta(t)$ is arbitrary function of the variable $t$ only.

\subsection{Translational symmetry}

In this case we take $a_{1}=0$. The characteristic equations corresponding to the translational symmetry are:

$$
\frac{d x}{a_{2}}=\frac{d y}{a_{3}}=\frac{d t}{a_{4}}=\frac{d u}{0}=\frac{d v}{0}=\frac{d p}{\delta(t)} .
$$

By solving the ODEs (11), we can obtain the similarity variables and similarity functions as follows:

$$
\begin{aligned}
& \phi=x-m_{1} t, \quad \psi=y-m_{2} t, \\
& u(x, y, t)=\hat{u}(\phi, \psi), \quad v(x, y, t)=\hat{v}(\phi, \psi), \quad p(x, y, t)=\hat{p}(\phi, \psi)+\delta_{1}(t),
\end{aligned}
$$

where $m_{1}=\frac{a_{2}}{a_{4}}, m_{2}=\frac{a_{3}}{a_{4}}$ are arbitrary constants and $\delta_{1}(t)=\int \delta(t) d t$ is an arbitrary function. Substituting the transformations (12), (13) in the Eq. (3) lead to the following system of PDEs:

$$
\left\{\begin{aligned}
E_{1}=\hat{u}_{\phi}+\hat{v}_{\psi}=0 & \\
E_{2}=\hat{u}[ & \left.\frac{1}{R k}+M \theta+\hat{u}_{\phi}-\lambda\left(m_{2} \hat{u}_{\phi \psi}+m_{1} \hat{u}_{\phi \phi}\right)\right]-\frac{1}{R}\left(\hat{u}_{\phi \phi}+\hat{u}_{\psi \psi}\right)+\lambda m_{2}^{2} \hat{u}_{\phi \phi}+\hat{p}_{\phi} \\
& -\hat{v}\left[m M \theta-\hat{u}_{\psi}+\lambda\left(m_{2} \hat{u}_{\psi \psi}+m_{1} \hat{u}_{\phi \psi}\right)\right]-m_{2}\left[\hat{u}_{\psi}\left(1+M \lambda \theta+\lambda \hat{v}_{\psi}+\lambda \hat{u}_{\phi}\right)\right. \\
& \left.+\lambda\left(-m M \theta \hat{v}_{\psi}-2 m_{1} \hat{u}_{\phi \psi}+\hat{p}_{\phi \psi}\right)\right]-m_{1}\left[\hat{u}_{\phi}\left(1+M \lambda \theta+\lambda \hat{u}_{\phi}\right)\right. \\
& \left.+\lambda\left(\hat{u}_{\psi} \hat{v}_{\phi}-m M \theta \hat{v}_{\phi}-m_{1} \hat{u}_{\phi \phi}+\hat{p}_{\phi \phi}\right)\right]=0 \\
E_{3}= & \hat{v}\left[\frac{1}{R k}+M \theta+\hat{v}_{\psi}-\lambda\left(m_{2} \hat{v}_{\psi \psi}+m_{1} \hat{v}_{\phi \psi}\right)\right]-\frac{1}{R}\left(\hat{v}_{\phi \phi}+\hat{v}_{\psi \psi}\right)-\lambda m_{2}^{2} \hat{v}_{\psi \psi}-\hat{p}_{\psi} \\
& +\hat{u}\left[m M \theta-\hat{v}_{\phi}-\lambda\left(m_{2} \hat{v}_{\phi \psi}+m_{1} \hat{v}_{\phi \phi}\right)\right]+m_{2}\left[\hat{v}_{\psi}\left(1+M \lambda \theta+\lambda \hat{v}_{\psi}\right)\right. \\
& \left.+\lambda\left(\hat{u}_{\psi}\left(m M \theta+\hat{v}_{\phi}\right)-2 m_{1} \hat{v}_{\phi \psi}+\hat{p}_{\psi \psi}\right)\right]+m_{1}\left[\hat{v}_{\phi}\left(1+M \lambda \theta+\lambda \hat{v}_{\psi}\right)\right. \\
& \left.+\lambda\left(\hat{u}_{\phi} \hat{v}_{\phi}+m M \theta \hat{u}_{\phi}+m_{1} \hat{v}_{\phi \phi}+\hat{p}_{\phi \psi}\right)\right]=0
\end{aligned}\right.
$$

To transform Eq. (14) to an (ODEs), we use the Lie group analysis again and obtain the infinitesimal generator corresponding to system of equation (14) in the following form:

$$
\xi_{11}=b_{1}, \quad \xi_{21}=b_{2}, \quad \eta_{11}=0, \quad \eta_{21}=0, \quad \eta_{31}=\beta(\phi, \psi),
$$

where $b_{i}, i=1,2$ are arbitrary constants and $\beta(\varphi, \psi)$ an arbitrary function that satisfy two conditions:

$$
\left\{\begin{array}{l}
\beta_{\phi}-\lambda\left(m_{2} \beta_{\phi \psi}+m_{1} \beta_{\phi \phi}\right)=0 \\
\beta_{\psi}-\lambda\left(m_{2} \beta_{\psi \psi}+m_{1} \beta_{\phi \psi}\right)=0
\end{array}\right.
$$


By solving the characteristic equations,

$$
\frac{d \phi}{b_{1}}=\frac{d \psi}{b_{2}}=\frac{d \hat{u}}{0}=\frac{d \hat{v}}{0}=\frac{d \hat{p}}{\beta(\phi, \psi)}
$$

if we take $\beta(\varphi, \psi)=0$ then we can obtain the similarity variable and similarity functions as the following:

$$
\chi=\psi-m_{0} \phi, \quad \hat{u}(\phi, \psi)=f(\chi), \hat{v}(\phi, \psi)=g(\chi), \hat{p}(\phi, \psi)=h(\chi),
$$

where $m_{0}=\frac{b_{2}}{b_{1}}$ an arbitrary constant. Substituting the transformations (18) in Eq. (14) lead to the following system of ODEs:

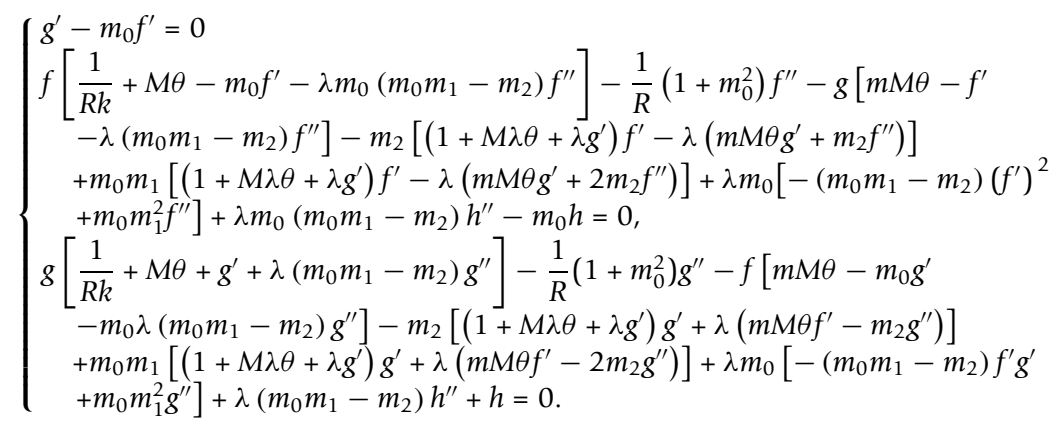

Integrating the first equation in (19) yields

$$
g=m_{0} f+c_{1},
$$

where $c_{1}$ is an arbitrary constant. Eliminating $h(\xi)$ from the second and third equations in (19) along with Eq. (20) we get the following equation:

$$
A f^{\prime \prime}+B f^{\prime}+C f+D=0
$$

where

$$
\begin{aligned}
A & =-\frac{1}{R}\left(1+m_{0}^{2}\right)+\lambda\left(m_{0}^{2} m_{1}^{2}+m_{2}^{2}-2 m_{0} m_{1} m_{2}+c_{1}\left(m_{0} m_{1}-m_{2}\right)\right), \\
B & =c_{1}+(1+M \lambda \theta)\left(m_{0} m_{1}-m_{2}\right), \\
C & =\frac{1}{R k}+M \theta \\
D & =\frac{c_{1}}{\left(1+m_{0}^{2}\right)}\left[m_{0}\left(\frac{1}{R k}+M \theta\right)-m M \theta\right] .
\end{aligned}
$$

By solving equation (21) we get

$$
f(\chi)=c_{2} \exp \left[\alpha_{1} \chi\right]+c_{3} \exp \left[\alpha_{2} \chi\right]-\tilde{D},
$$

where $\tilde{D}=\frac{D}{C}$ and $c_{2}, c_{3}$ are arbitrary constants and $\alpha_{1}, \alpha_{2}$ are roots of the following equation:

$$
A \gamma^{2}+B \gamma+C=0
$$


From equations (20) and (23) the expression of the function $g(\chi)$ becomes

$$
g(\chi)=m_{0}\left(c_{2} \exp \left[\alpha_{1} \chi\right]+c_{3} \exp \left[\alpha_{2} \chi\right]-\tilde{D}+c_{1}\right.
$$

and from the second and third equations in (19) we get

$$
\begin{aligned}
h(\chi)= & {\left[\tilde{D} m M \theta-\frac{c_{1}}{\left(1+m_{0}^{2}\right)}\left(\frac{1}{R k}+M \theta+m M \theta m_{0}\right)\right] \chi-m M \theta\left(\frac{c_{2}}{\alpha_{1}} \exp \left[\alpha_{1} \chi\right]\right.} \\
& \left.+\frac{c_{3}}{\alpha_{2}} \exp \left[\alpha_{2} \chi\right]\right)-\lambda c_{4}\left(m_{0} m_{1}-m_{2}\right) \exp \left[-\frac{\chi}{\lambda\left(m_{0} m_{1}-m_{2}\right)}\right]+c_{5},
\end{aligned}
$$

where $c_{4}$ and $c_{5}$ are arbitrary constants.

In the form of the original variables, our exact solutions can be written as follows:

$$
\left\{\begin{aligned}
u(x, y, t)= & c_{2} \exp \left[\alpha_{1}\left(y-m_{0} x+\left(m_{0} m_{1}-m_{2}\right) t\right)\right] \\
& +c_{3} \exp \left[\alpha_{2}\left(y-m_{0} x+\left(m_{0} m_{1}-m_{2}\right) t\right)\right]-\tilde{D}, \\
v(x, y, t)= & m_{0}\left(c_{2} \exp \left[\alpha_{1}\left(y-m_{0} x+\left(m_{0} m_{1}-m_{2}\right) t\right)\right]\right. \\
& \left.+c_{3} \exp \left[\alpha_{2}\left(y-m_{0} x+\left(m_{0} m_{1}-m_{2}\right) t\right)\right]-\tilde{D}\right)+c_{1}, \\
p(x, y, t)= & {\left[\tilde{D} m M \theta-\frac{c_{1}}{\left(1+m_{0}^{2}\right)}\left(\frac{1}{R k}+M \theta+m M \theta m_{0}\right)\right]\left(y-m_{0} x+\left(m_{0} m_{1}-m_{2}\right)\right) } \\
& -m M \theta\left(\frac{c_{2}}{\alpha_{1}} \exp \left[\alpha_{1}\left(y-m_{0} x+\left(m_{0} m_{1}-m_{2}\right) t\right)\right]\right. \\
& \left.+\frac{c_{3}}{\alpha_{2}} \exp \left[\alpha_{2}\left(y-m_{0} x+\left(m_{0} m_{1}-m_{2}\right) t\right)\right]\right) \lambda \\
& -c_{4}\left(m_{0} m_{1}-m_{2}\right) \exp \left[-\frac{\left(y-m_{0} x+\left(m_{0} m_{1}-m_{2}\right) t\right)}{\lambda\left(m_{0} m_{1}-m_{2}\right)}\right]+c_{5}+\delta_{1}(t) .
\end{aligned}\right.
$$

\subsection{Rotational symmetry}

In this section, the parameter $a_{1}$ is taken to be an arbitrary non-zero constant. The characteristic equations corresponding to the rotational symmetry are:

$$
\frac{d x}{-a_{1} y+a_{2}}=\frac{d y}{a_{1} x+a_{3}}=\frac{d t}{a_{4}}=\frac{d u}{-a_{1} v}=\frac{d v}{a_{1} u}=\frac{d p}{\delta(t)} .
$$

Integrating equations (28) using the Lie group analysis method, we get the rotationally invariant solutions for our problem in the following form:

$$
\left\{\begin{array}{l}
\psi=\sqrt{\left(x+\beta_{1}\right)^{2}+\left(y+\beta_{2}\right)^{2}} \\
\phi=\tan ^{-1}\left[\frac{\gamma+\beta_{2}}{x+\beta_{1}}\right] \\
u=G_{1}(\psi, t) \cos [\phi]+G_{2}(\psi, t) \sin [\phi] \\
v=G_{1}(\psi, t) \sin [\phi]-G_{2}(\psi, t) \cos [\phi] \\
p=G_{3}(\psi, t)+\delta_{1}(t)
\end{array}\right.
$$

where $\beta_{1}=\frac{a_{3}}{a_{1}}, \beta_{2}=\frac{-a_{2}}{a_{1}}$, and $G_{3}$ are functions of $\psi$ and $t$.

Substituting the new (similarity) variables $(\varphi, \psi)$ and functions $\left(G_{1}, G_{2}, G_{3}\right)$ into the original system (3) yields the following set of equations: 


$$
\left\{\begin{array}{l}
\frac{G_{1}}{\psi}+\frac{\partial G_{1}}{\partial \psi}=0 \\
G_{1}\left[k+\psi^{2}\left(1+R k M \theta\left(\frac{\partial G_{1}}{\partial \psi}+\lambda \frac{\partial^{2} G_{1}}{\partial \psi \partial t}\right)\right)\right]+k \psi\left[R G_{2}\left(m M \theta \psi-G_{2}-2 \lambda \frac{\partial G_{2}}{\partial t}\right)-\frac{\partial G_{1}}{\partial \psi}\right] \\
\quad-k \psi^{2} \frac{\partial^{2} G_{1}}{\partial \psi^{2}}+R k \psi^{2}\left[\left(1+M \lambda \theta+\lambda \frac{\partial G_{1}}{\partial \psi}\right) \frac{\partial G_{1}}{\partial t}+\frac{\partial G_{3}}{\partial \psi}\right. \\
\left.+\lambda\left(m M \theta \frac{\partial G_{2}}{\partial t}+\frac{\partial^{2} G_{1}}{\partial t^{2}}+\frac{\partial^{2} G_{3}}{\partial \psi}\right)\right]=0 \\
G_{2}\left[k+\psi^{2}(1+R k M \theta)+R k \lambda \psi \frac{\partial G_{1}}{\partial t}\right]+k \psi\left[R G_{1}\left(G_{2}+\lambda \frac{\partial G_{2}}{\partial t}\right)-\frac{\partial G_{2}}{\partial \psi}-\psi \frac{\partial^{2} G_{2}}{\partial \psi^{2}}\right] \\
+R k \psi^{2}\left[(1+M \lambda \theta) \frac{\partial G_{2}}{\partial t}+\lambda\left(\frac{\partial^{2} G_{2}}{\partial t^{2}}+\frac{\partial G_{1}}{\partial t} \frac{\partial G_{2}}{\partial \psi}+G_{1} \frac{\partial^{2} G_{2}}{\partial \psi \partial t}\right)\right. \\
\left.\quad-m M \theta G_{1}-m M \lambda \theta \frac{\partial G_{1}}{\partial t}+G_{1} \frac{\partial G_{2}}{\partial \psi}\right]=0
\end{array}\right.
$$

To transform Eq. (30) to (ODEs), we use the Lie group analysis again and obtain the infinitesimal generator corresponding to system of equations (30) in the following form:

$$
\xi_{12}=0, \quad \xi_{22}=d_{1}, \quad \eta_{11}=0, \quad \eta_{21}=0, \quad \eta_{32}=\eta\left(\psi, t, G_{1}\right),
$$

where $d_{1}$ is an arbitrary constant and $\eta\left(\psi, t, G_{1}\right)$ an arbitrary function that satisfy the following condition:

$$
\psi \frac{\partial \eta}{\partial \psi}-G_{1} \frac{\partial \eta}{\partial G_{1}}=0 .
$$

If we take $\eta\left(\psi, t, G_{1}\right)=0$ is a simple solution of Eq. (32) then the characteristic equations are:

$$
\frac{d \psi}{0}=\frac{d t}{d_{1}}=\frac{d G_{1}}{0}=\frac{d G_{2}}{0}=\frac{d G_{3}}{0} .
$$

The similarity variables and resulting functions are

$$
\zeta=\psi, G_{1}=F_{1}(\zeta), G_{2}=F_{2}(\zeta), G_{3}=F_{3}(\zeta)
$$

One now substitutes the similarity variable and the functions into the equations (30) and obtains

$$
\left\{\begin{array}{l}
F_{1}^{\prime}+\frac{F_{1}}{\zeta}=0 \\
F_{1}\left[k+\zeta^{2}\left(1+R k M \theta+R k F_{1}^{\prime}\right)\right]+k \zeta\left[R F_{2}\left(m M \theta \zeta-F_{2}\right)-F_{1}^{\prime}+R \zeta F^{\prime}{ }_{3}-\zeta F^{\prime \prime}{ }_{1}\right]=0 \\
F_{2}\left[k+\zeta^{2}(1+R k M \theta)\right]+R k \zeta F_{1}\left[F_{2}+\zeta\left(F_{2}^{\prime}-m M \theta\right)\right]-k \zeta F^{\prime}{ }_{2}+\zeta F^{\prime \prime}{ }_{2}=0
\end{array}\right.
$$

Integrating the first equation in the system (35) we get:

$$
F_{1}=\frac{g_{1}}{\zeta}
$$

where $g_{1}$ is an arbitrary constant. From the second and the third equations in (35) along with (36) we get:

$$
\begin{aligned}
& F_{3}^{\prime}-\frac{F_{2}^{2}}{\zeta}+m M \theta F_{2}-\frac{g_{1}^{2}}{\zeta^{3}}+\frac{(1+R k M \theta) g_{1}}{R k \zeta}=0, \\
& F_{2}\left[k+\zeta^{2}(1+R k M \theta)\right]+R k g_{1}\left[F_{2}+\zeta\left(F_{2}^{\prime}-m M \theta\right)\right]-k \zeta\left[F_{2}^{\prime}+\zeta F^{\prime \prime}{ }_{2}\right]=0,
\end{aligned}
$$


by integrating equation (38) we obtain

$$
\begin{aligned}
& F_{2}=\frac{1}{k} 2^{-\left(1+\frac{R g_{1}}{2}\right)_{\zeta} \frac{R g_{1}}{2}}\left(-i \zeta \sqrt{\frac{1}{k}+R M \theta}\right)^{-\left(1+\frac{R g_{1}}{2}\right)} \\
& {\left[2^{2+R_{g_{1}} k g_{3} \csc }\left[\frac{1}{2} \pi R g_{1}\right]{ }_{0} F_{1} \text { Regularized }\left(-\frac{R g_{1}}{2}, \frac{\zeta^{2}(1+R k M \theta)}{4 k}\right)\right.} \\
& -\zeta^{2}\left(-i \zeta \sqrt{\frac{1}{k}+R M \theta}\right)(1+R k M \theta)\left[g_{2}+g_{3} \cot \left[\frac{1}{2} \pi R g_{1}\right]\right] \\
& \left.{ }_{0} F_{1} \text { Regularized }\left(2+\frac{R g_{1}}{2}, \frac{\zeta^{2}(1+R k M \theta)}{4 k}\right)\right] \\
& -\frac{R k m M \theta g_{1}}{\zeta(1+R k M \theta)}\left[-1+{ }_{0} F_{1}\left(-\frac{R g_{1}}{2}, \frac{\zeta^{2}(1+R k M \theta)}{4 k}\right)\right] \text {, }
\end{aligned}
$$

and

$$
F_{3}=\int\left(\frac{F_{2}^{2}}{\zeta}-m M \theta F_{2}+\frac{g_{1}^{2}}{\zeta^{3}}-\frac{(1+R k M \theta) g_{1}}{R k \zeta}\right) d \zeta,
$$

where $g_{2}$ and $g_{3}$ are arbitrary constants, and the function ${ }_{p} F_{q}$ and $F_{p}$ Regularized are defined as:

$$
\begin{aligned}
& \begin{array}{l}
{ }_{p} F_{q}(\vec{a} ; \vec{b} ; z)=\text { HypergeometricPFQ }\left(\left\{a_{1}, \ldots, a_{p}\right\} ;\left\{b_{1}, \ldots, b_{q}\right\} ; z\right) \\
\quad=\sum_{k=0}^{\infty}\left[\frac{\left(a_{1}\right) k \ldots\left(a_{p}\right) k}{\left(b_{1}\right) k \ldots\left(b_{q}\right) k}\right] \frac{z^{k}}{k !},
\end{array} \\
& { }_{p} F_{q} \text { Regularized }=\frac{{ }_{p} F_{q}}{\Gamma\left(b_{1}\right) \ldots \Gamma\left(b_{q}\right)} .
\end{aligned}
$$

Then the solution of our problem in the original variables using symbolic computations is:

$$
\begin{aligned}
& u(x, y, t)=\frac{g_{1}}{\psi} \cos [\phi]+\left\{\frac{1}{k} 2^{-\left(1+\frac{R g_{1}}{2}\right)} \psi \frac{R g_{1}}{2}\left(-i \psi \sqrt{\frac{1}{k}+R M \theta}\right)^{-\left(1+\frac{R g_{1}}{2}\right)}\right. \\
& {\left[2^{2+R g_{1}} \mathrm{~kg}_{3} \csc \left[\frac{1}{2} \pi R g_{1}\right]_{0} F_{1} \text { Regularized }\left(-\frac{R g_{1}}{2}, \frac{\psi^{2}(1+R k M \theta)}{4 k}\right)-\right.} \\
& \psi^{2}\left(-i \psi \sqrt{\frac{1}{k}+R M \theta}\right)(1+R k M \theta)\left[g_{2}+g_{3} \cot \left[\frac{1}{2} \pi R g_{1}\right]\right] \\
& \left.{ }_{0} F_{1} \text { Regularized }\left(2+\frac{R g_{1}}{2}, \frac{\psi^{2}(1+R k M \theta)}{4 k}\right)\right]- \\
& \left.\frac{R k m M \theta g_{1}}{\psi(1+R k M \theta)}\left[-1+_{0} F_{1}\left(-\frac{R g_{1}}{2}, \frac{\psi^{2}(1+R k M \theta)}{4 k}\right)\right]\right\} \sin [\phi] \\
& v(x, y, t)=\frac{g_{1}}{\psi} \sin [\phi]-\left\{\frac{1}{k} 2^{-\left(1+\frac{R g_{1}}{2}\right)} \psi \frac{R g_{1}}{2}\left(-i \psi \sqrt{\frac{1}{k}+R M \theta}\right)^{-\left(1+\frac{R g_{1}}{2}\right)}\right. \\
& {\left[2^{2+R_{g 1}} \mathrm{~kg}_{3} \csc \left[\frac{1}{2} \pi R g_{1}\right]_{0} F_{1} \text { Regularized }\left(-\frac{R g_{1}}{2}, \frac{\psi^{2}(1+R k M \theta)}{4 k}\right)-\right.} \\
& -\psi^{2}\left(-i \psi \sqrt{\frac{1}{k}+R M \theta}\right)(1+R k M \theta)\left[g_{2}+g_{3} \cot \left[\frac{1}{2} \pi R g_{1}\right]\right] \\
& \left.{ }_{0} F_{1} \text { Regularized }\left(2+\frac{R g_{1}}{2}, \frac{\psi^{2}(1+R k M \theta)}{4 k}\right)\right]- \\
& \left.\frac{R k m M \theta g_{1}}{\psi(1+R k M \theta)}\left[-1+{ }_{0} F_{1}\left(-\frac{R g_{1}}{2}, \frac{\psi^{2}(1+R k M \theta)}{4 k}\right)\right]\right\} \cos [\phi], \\
& p(x, y, t)=\int\left(\frac{F_{2}^{2}}{\psi}-m M \theta F_{2}+\frac{g_{1}^{2}}{\psi^{3}}-\frac{(1+R k M \theta) g_{1}}{R k \psi}\right) d \psi+\delta_{1}(t)
\end{aligned}
$$


where $\psi$ and $\varphi$ are the same in (29).

\section{Solutions for hydro-magnetic Maxwell fluid through a porous medium: (magmatic fluid) problem}

One of the important applications in geology is the magmatic fluid. Consider a magmatic fluid as an incompressible hydro-magnetic Maxwell fluid through a porous medium and a plate over it. The plate occupies the position $y=0$, where the positive $y$ goes deep into the fluid beneath the plate. The relevant boundary conditions are of the form:

$$
\begin{aligned}
& u(x, 0,0)=U_{0}, \quad u(x, \infty, t)=0, \quad \frac{\partial u}{\partial y}(x, 0,0)=0, \\
& v(x, 0,0)=-V_{0}, \quad p(x, \infty, 0)=P_{0}, \quad p(x, 0,0)=P_{a},
\end{aligned}
$$

where $U_{0}$ is the velocity of the plate, $V_{0}$ is the magmatic fluid velocity penetrating into the plate, $P_{0}$ is the pressure deep in the magmatic fluid and $P_{a}$ is the atmosphere pressure. The expressions (27) for the translational symmetry case solution after using conditions (44) give

$$
\left\{\begin{aligned}
u(x, y, t)= & \frac{U_{0}}{\alpha_{2}-\alpha_{1}}\left[\alpha_{2} \exp \left[\alpha_{1}(y+W t)\right]-\alpha_{1} \exp \left[\alpha_{2}(y+W t)\right]\right] \\
v(x, y, t)= & \frac{V_{0}}{\alpha_{2}-\alpha_{1}}\left[\alpha_{2} \exp \left[\alpha_{1}(y+W t)\right]-\alpha_{1} \exp \left[\alpha_{2}(y+W t)\right]\right] \\
p(x, y, t)= & \frac{m M \theta U_{0}}{\alpha_{1} \alpha_{2}\left(\alpha_{2}-\alpha_{1}\right)}\left[-\alpha_{1}^{2} \exp \left[\alpha_{2}(y+W t)\right]+\alpha_{2}^{2} \exp \left[\alpha_{1}(y+W t)\right]\right] \\
& \quad-\left[p_{0}-p_{a}+\frac{m M \theta U_{0}}{\alpha_{1} \alpha_{2}}\left(\alpha_{1}+\alpha_{2}\right)\right] \exp \left[-\frac{(y+W t)}{C \lambda}\right]+P_{0},
\end{aligned}\right.
$$

where $W=m_{0} m_{1}-m_{2}, \alpha_{1}$ and $\alpha_{2}$ are the negative roots of Eq. (24).

\section{Discussion of the magmatic fluid problem}

This section deals with the graphics on the magmatic fluid. So, the interpretation of the relaxation time $\lambda$, Reynolds number $R$, Hartmann number $M$, Hall parameter $m$, the time parameter $t$, and the permeability parameter $k$ have been studied on the pressure $p$, and the $x$ and $y$ components of the velocity distributions $u$ and $v$.

Figures $1,2,3,4,5,6,7,8,9,10,11,12,13$, and 14 describe the variations of the velocity components $u$ and $v$ with the time $t$ at $y=0$ for different values of

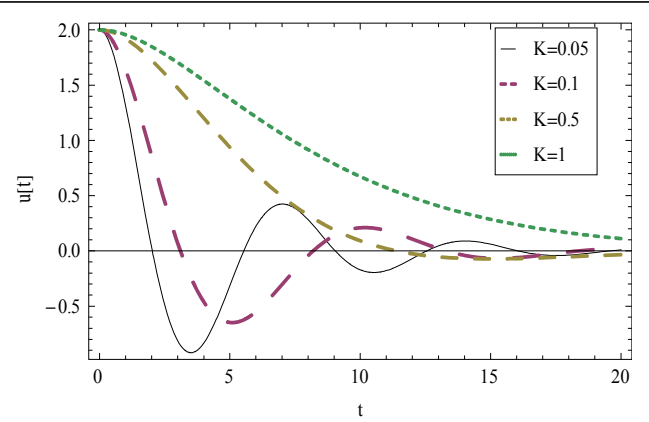

Figure 1 Variation of the dimensionless velocity distribution along the $x$-axis with $t$ for different values of permeability parameter $k\left(U_{0}=V_{0}=2 ; m_{0}=m_{1}=m_{2}=2 ; y=0 ; m=0.5 ; M=0.5 ; R=\right.$ $0.5 ; \lambda=50)$. 


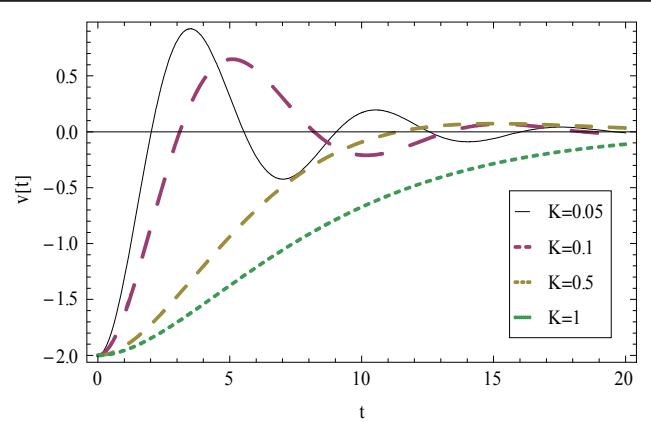

Figure 2 Variation of the dimensionless velocity distribution along the $y$-axis with $t$ for different values of permeability parameter $k\left(U_{0}=V_{0}=2 ; m_{0}=m_{1}=m_{2}=2 ; y=0 ; m=0.5 ; M=0.5 ; R=\right.$ $0.5 ; \lambda=50$ ).

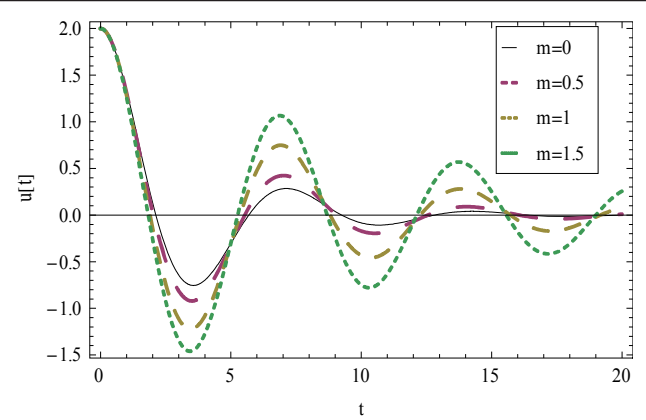

Figure 3 Variation of the dimensionless velocity distribution along the $x$-axis with $t$ for different values of Hall parameter $m\left(U_{0}=V_{0}=2 ; y=0 ; m_{0}=m_{1}=m_{2}=2 ; k=0.05 ; M=0.5 ; R=0.5 ; \lambda=50\right.$ ).

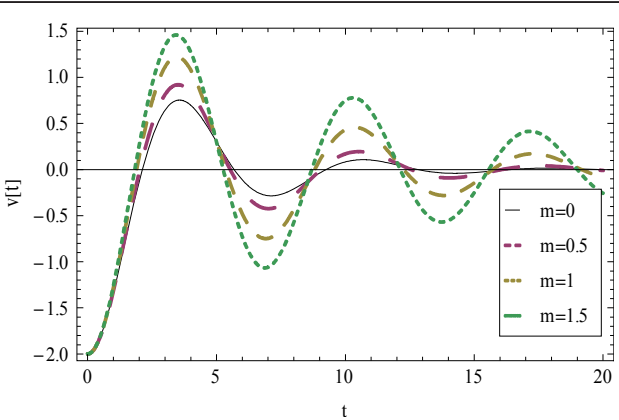

Figure 4 Variation of the dimensionless velocity distribution along the $y$-axis with $t$ for different values of Hall parameter $m\left(U_{0}=V_{0}=2 ; y=0 ; m_{0}=m_{1}=m_{2}=2 ; k=0.05 ; M=0.5 ; R=0.5 ; \lambda=50\right.$ ).

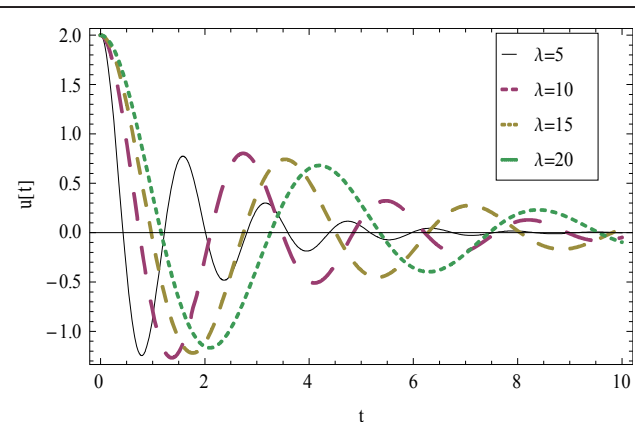

Figure 5 Variation of the dimensionless velocity distribution along the $x$-axis with $t$ for different values of relaxation time $\lambda\left(U_{0}=V_{0}=2 ; y=0 ; m_{0}=m_{1}=m_{2}=2 ; k=0.05 ; M=0.5 ; R=0.5 ; m=0.5\right)$. 


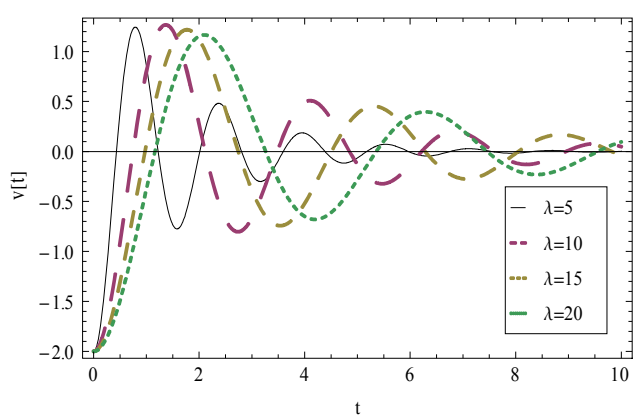

Figure 6 Variation of the dimensionless velocity distribution along the $y$-axis with $t$ for different values of relaxation time $\lambda\left(U_{0}=V_{0}=2 ; y=0 ; m_{0}=m_{1}=m_{2}=2 ; k=0.05 ; M=0.5 ; R=0.5 ; m=0.5\right.$ ).

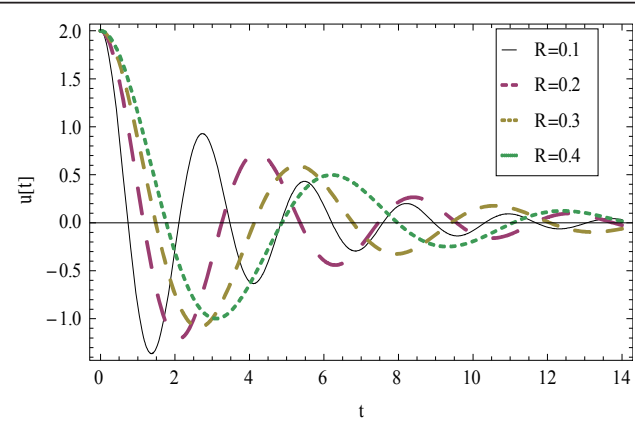

Figure 7 Variation of the dimensionless velocity distribution along the $x$-axis with $t$ for different values of Reynolds number $R\left(U_{0}=V_{0}=2 ; y=0 ; m=0.5 ; m_{0}=m_{1}=m_{2}=2 ; k=0.05 ; M=0.5 ; \lambda=50\right)$.

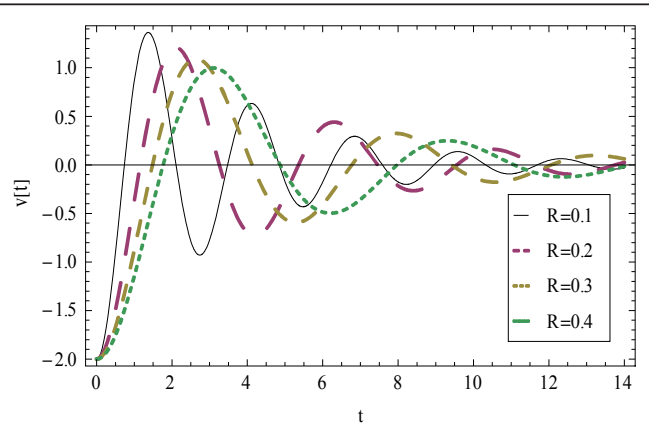

Figure 8 Variation of the dimensionless velocity distribution along the $y$-axis with $t$ for different values of Reynolds number $R\left(U_{0}=V_{0}=2 ; y=0 ; m=0.5 ; m_{0}=m_{1}=m_{2}=2 ; k=0.05 ; M=0.5 ; \lambda=50\right)$.

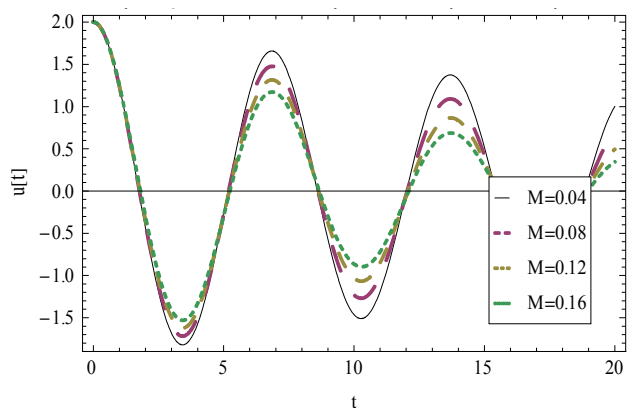

Figure 9 Variation of the dimensionless velocity distribution along the $x$-axis with $t$ for different values of Hartmann number $M\left(U_{0}=V_{0}=2 ; m_{0}=m_{1}=m_{2}=2 ; y=0 ; k=0.05 ; R=0.5 ; \lambda=50 ; m=0.5\right)$. 


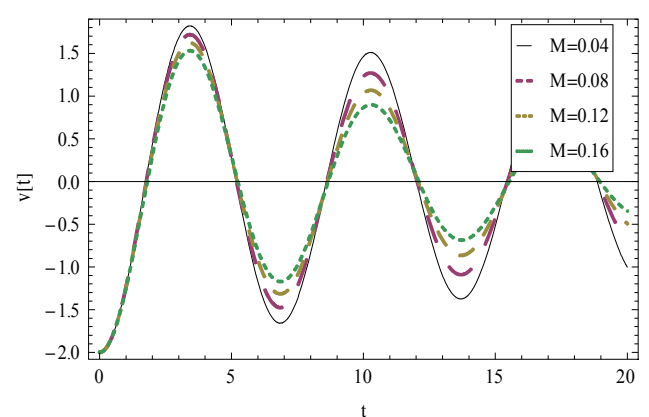

Figure 10 Variation of the dimensionless velocity distribution along the $y$-axis with $t$ for different values of Hartmann number $M\left(U_{0}=V_{0}=2 ; m_{0}=m_{1}=m_{2}=2 ; y=0 ; k=0.05 ; R=0.5 ; \lambda=50 ; m=0.5\right)$.

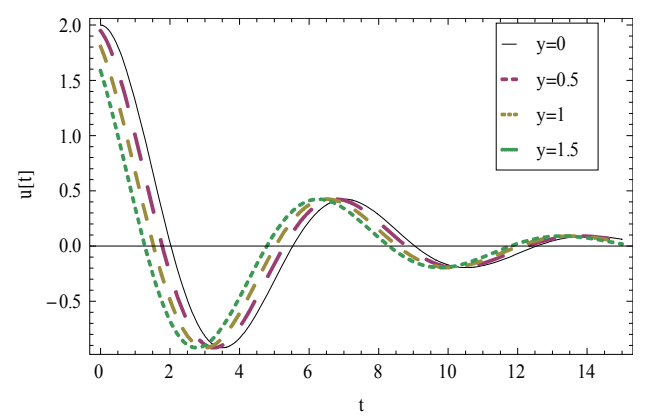

Figure 11 Variation of the dimensionless velocity distribution along the $x$-axis with $t$ for different values $y\left(U_{0}=V_{0}=2 ; m_{0}=m_{1}=m_{2}=2 ; M=0.5 ; k=0.05 ; R=0.5 ; \lambda=50 ; m=0.5\right)$

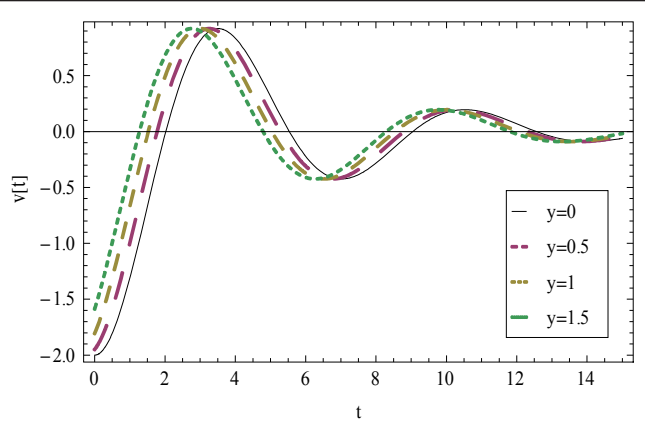

Figure 12 Variation of the dimensionless velocity distribution along the $y$-axis with $t$ for different values $y\left(U_{0}=V_{0}=2 ; m_{0}=m_{1}=m_{2}=2 ; M=2 ; k=0.05 ; R=50 ; \lambda=50 ; m=0.5\right)$.

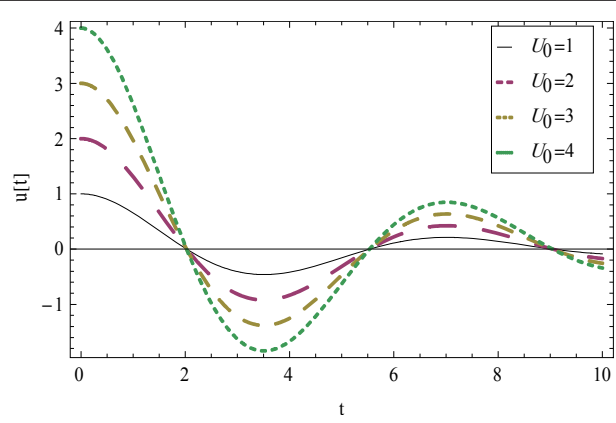

Figure 13 Variation of the dimensionless velocity distribution along the $x$-axis with $t$ for different values of the velocity $U_{0}\left(V_{0}=2 ; y=0 ; m_{0}=m_{1}=m_{2}=2 ; k=0.05 ; R=0.5 ; \lambda=50 ; m=0.5 ; M=0.5\right.$ ). 


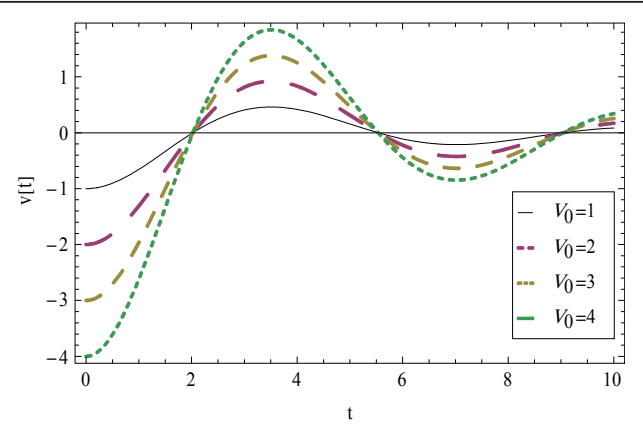

Figure 14 Variation of the dimensionless velocity distribution along the $y$-axis with $t$ for different values of magmatic velocity $V_{0}\left(U_{0}=2 ; y=0 ; m_{0}=m_{1}=m_{2}=2 ; k=0.05 ; R=0.5 ; \lambda=50 ; m=0.5\right.$; $M=0.5)$

permeability parameter $k$, Hall parameter $m$, the relaxation time $\lambda$, Reynolds number R, and Hartmann number $M$. For all of these figures at $y=0$ we note that as the time $t$ increases the variation of each component of the velocity decreases and vanishes for large values of $t$. This is expected, where for small values of $t$ and at the magma plate $(y=0)$, the variation of the velocity components is obvious. Also, the gap between the curves for small values of $t$ at the magma plate increases than those as $t$ increases.

Figures 1 and 2 show that as the permeability parameter $k$ increases the horizontal velocity component $u$ increases, while the vertical velocity component $v$ decreases. Figures 3 and 4 illustrate the variation of the velocity components $u$ and $v$ with the Hall parameter $m$, which indicate that for small values of $t$ (or at initial values of $t$ ) the curves are the same with no obvious different which for $t>2$, the gap between the curves appears. Also, we can see that curves with small values of $m(m=0,0.5)$ are vanishing rabidly than those for $(m=1,1.5)$ i.e., as the Hall parameter $m$ increases the disturbance of the velocity components increase. (decreasing the number of density electrons or the electronic charges).

Figures 5 and 6 illustrate the variations of $u$ and $v$ with $t$ for different values of the relaxation time $\lambda$, which show that for small values $\lambda$ the disturbance in $u$ and $v$ will vanish rapidly than those as $\lambda$ increases. Also, the figures show that the disturbance in $u$ and $v$ for a Newtonian fluid less than those for a Non-Newtonian fluid in the case of magma flow.

Figures 7 and 8 show that the variation with the Reynolds number $R$. As $R$ increases the velocity components $u$ and $v$ increase. Figures 9 and 10 show that as the Hartmann number $M$ increases the velocity components $u$ and $v$ decrease, i.e., the fluid moves as a block and takes a constant value for large values of $M$. Figures 11 and 12 illustrate the variation of $u$ and $v$ with $t$ for different values of the $y$ axis, which show that the velocity components take the initial values of the magma plate at $y=0$ and the velocity components decreases as $y$ increases. Figures 13 and 14 describe the variations of $u$ and $v$ with $t$ for different values of $U_{0}$ and $\mathrm{V}_{0}$ (velocities of the magma plate), the figures show that the gab between the curves decreases with time and finally vanishes and for certain values of $t$ the velocity components $u$ and $v$ equal to zero. Also, the magnitudes of $u$ and $v$ increase with increasing $U_{0}$ and $V_{0}$.

Figures 15, 16, 17, 18, 19, and 20 illustrate the variation of the pressure $p$ with $y$ for different values of $k, m, \lambda, R, M$, and $t$. We can see that the pressure decreases as the 


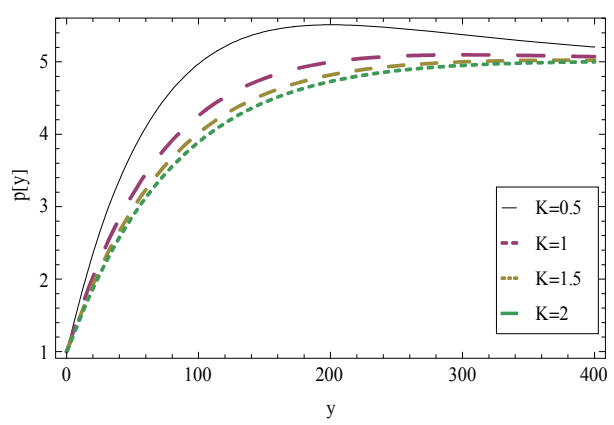

Figure 15 Variation of the dimensionless pressure distribution with $y$ for different values of permeability parameter $k\left(U_{0}=V_{0}=2 ; p_{0}=5 ; p a=1 ; m_{0}=m_{1}=m_{2}=2 ; t=0 ; m=0.5 ; M=2 ; R=\right.$ $50 ; \lambda=50)$

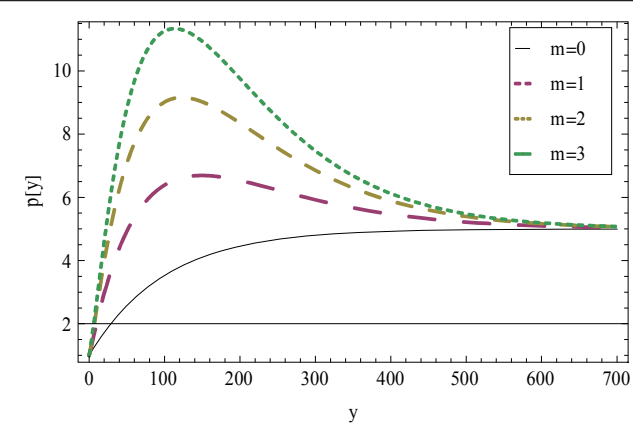

Figure 16 Variation of the dimensionless pressure distribution with $y$ for different values of Hall parameter $m\left(U_{0}=V_{0}=2 ; m_{0}=m_{1}=m_{2}=2 ; t=0 ; k=0.5 ; M=2 ; R=50 ; \lambda=50 ; p_{0}=5 ; p_{a}=1\right)$.

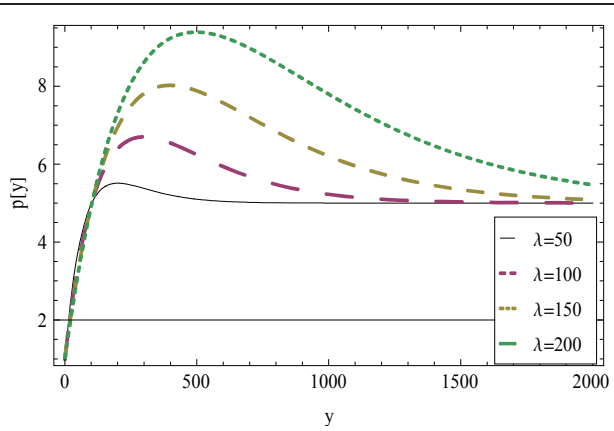

Figure 17 Variation of the dimensionless pressure distribution with $y$ for different values of relaxation time $\lambda\left(U_{0}=V_{0}=2 ; m_{0}=m_{1}=m_{2}=2 ; t=0 ; k=0.5 ; M=2 ; R=50 ; m=0.5 ; p_{0}=5 ; p_{a}=1\right)$.

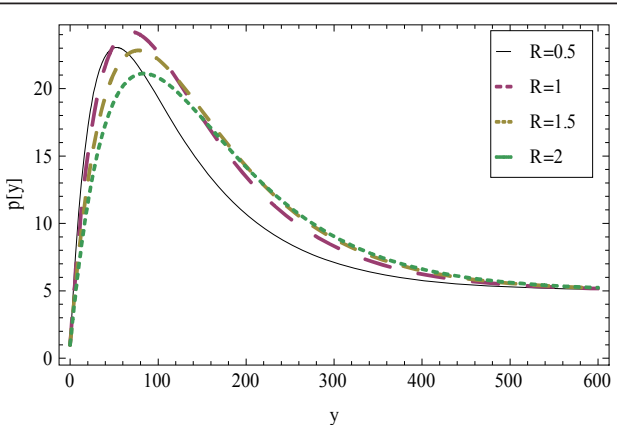

Figure 18 Variation of the dimensionless pressure distribution with $y$ for different values of Reynolds number $R\left(U_{0}=V_{0}=2 ; m_{0}=m_{1}=m_{2}=2 ; t=0 ; k=0.5 ; M=2 ; \lambda=50 ; m=0.5 ; p_{0}=5 ; p_{a}=1\right)$ 


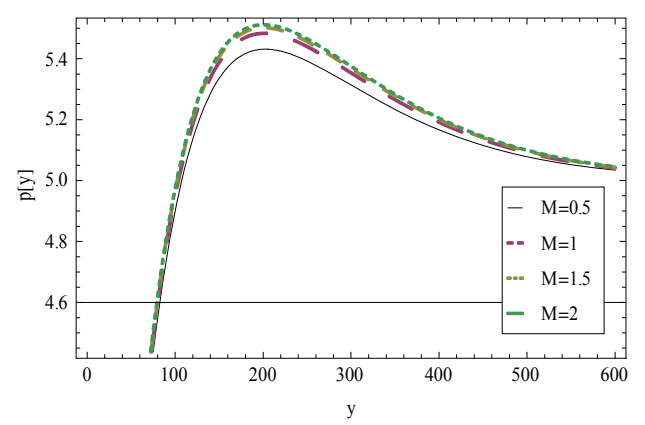

Figure 19 Variation of the dimensionless pressure distribution with $y$ for different values of Hartmann number $M\left(U_{0}=V_{0}=2 ; m_{0}=m_{1}=m_{2}=2 ; t=0 ; k=0.5 ; R=50 ; \lambda=50 ; m=0.5 ; p_{0}=5\right.$; $\left.p_{a}=1\right)$

permeability parameter $k$ increases and take a constant value for large values of $k$. However an inverse effective behavior for $p$ with the Hartmann number $M$ is shown in Figure 19. Figures 16 and 17 describe the variation of $p$ with the Hall parameter $m$ and the relaxation time $\lambda$, which shows that as $y$ increases the pressure increases and the gabs between the curves are more obvious near to the magma plate. Also, the pressure takes the same values of the pressure deep in the magmatic fluid $P_{0}$ for the large values of $y$ (as we move deep into the fluid) and the same effect is shown with $\lambda$. The pressure decreases as the Reynolds number $R$ increases as shown in Figure 18. Figure 20 shows the variation of the pressure with $y$ for different values of $t$. We can see that the pressure increases as $t$ increases for small values of $y$.

Other cases of symmetry will be considered for other boundary value problems else where for other applications.

\section{Concluding remarks}

The significant features of Lie group analysis for hydro-magnetic Maxwell fluid through a porous medium have been presented. Similarities solutions are obtained and applied to an important phenomena in geology, which is the magmatic fluid. The main points have been summarized as follows:

-As the Hall parameter $m$ increases the disturbances of the velocity components are increase.

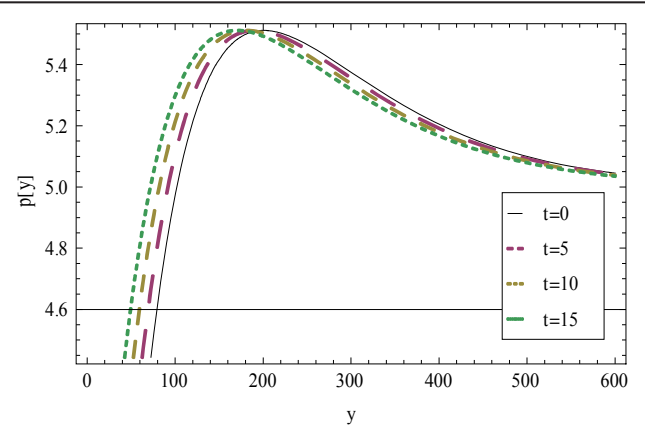

Figure 20 Variation of the dimensionless pressure distribution with $y$ for different values of time $t$ $\left(U_{0}=V_{0}=2 ; m_{0}=m_{1}=m_{2}=2 ; M=2 ; k=0.5 ; R=50 ; \lambda=50 ; m=0.5 ; p_{0}=5 ; p_{a}=1 ; y=0\right)$. 
- The disturbances in the fluid velocity components for a Newtonian fluid are less than those for a non-Newtonian fluid (magmatic fluid)

- The magmatic fluid moves as a block for large values of the Hartmann number $M$. - The pressure near to the magma plate is higher for a magneto-magma flow than that for a magma flow without a magnetic field. Also, this pressure for a porous medium is less than that for a medium with high permeability.

- The pressure increase near to the magma plate and take the constant value of the pressure deep in the magmatic fluid for large values of $y$.

\author{
Author details \\ ${ }^{1}$ Mathematics Department, Faculty of Science, Al-Azhar University, Nasr City 11448, Cairo, Egypt ${ }^{2}$ Mathematics \\ Department, Faculty of Science, Minia University, Minia 61111, Egypt
}

\title{
Authors' contributions
}

Khaled S. Mekheimer, Mostafa F El-Sabbagh and and Rabea E. Abo-Elkhair contributed to each part of this work equally.

All the authors read and approved the final manuscript.

\section{Competing interests}

The authors declare that they have no competing interests.

Received: 20 August 2011 Accepted: 13 February 2012 Published: 13 February 2012

\section{References}

1. Zhaosheng, $Y$, Jianzhong, L: Numerical research on the coherent structure in the viscoelastic second-order mixing layers. Appl Math Mech. 19, 671-677 (1998)

2. Shifang, H: Constitutive Equation and Computational Analytical Theory of Non-Newtonian Fluids. Science Press, Beijing (2000)

3. Zhaosheng, JE, Rajagopal, KR: Fluids of differential type: critical review and thermodynamic analysis. Int J Eng Sci. 33, 689-729 (1995). doi:10.1016/0020-7225(94)00078-X

4. Aksel, N: A brief note from the editor on the second-order fluid. Acta. Mech. 157, 235-236 (2002). doi:10.1007/ BF01182167

5. Maxwell, JC: On the dynamical theory of gases. Philos Trans R Soc Lond A. 157, 26-78 (1866)

6. Fetecau, C, Fetecau, C: Decay of a potential vortex in a Maxwell fluid. Int J Nonlinear Mech. 38, $985-990$ (2003). doi:10.1016/S0020-7462(02)00042-2

7. Fetecau, C, Zierep, J, Angew, Z: The RayleighStokes-problem for a Maxwell fluid. Math Phys. 54, 1086-1093 (2003)

8. Hayat, T, Nadeem, S, Asghar, S: Periodic unidirectional flows of a vis-coelastic fluid with fractional Maxwell model. Appl Math Comput. 151, 153-161 (2004). doi:10.1016/S0096-3003(03)00329-1

9. Tan, WC, Pan, WX, Xu, MY: A note on unsteady flows of a viscoelastic fluid with the fractional Maxwell model between two parallel plates. Int J Nonlinear Mech. 38, 645-650 (2003). doi:10.1016/50020-7462(01)00121-4

10. Dagan, G: Flow and Transport in Porous Formations. Springer-Verlag, Berlin (1989)

11. Ali, AT: A note on the Exp-function method and its application to nonlinear equations. Phys Scr 79 (2009). 025006

12. El-Sabbagh, MF, Ali, AT: New generalized Jacobi elliptic function expansion method. Commun Nonl Sci Numer Simul. 13, 1758-1766 (2008). doi:10.1016/j.cnsns.2007.04.014

13. Ali, AT: New generalized Jacobi elliptic function rational expansion method. J Comput Appl Math. 235(14), 4117-4127 (2011). doi:10.1016/j.cam.2011.03.002

14. Ali, AT: New exact solutions of Einstein vacuum equations for rotating axially symmetric fields. Phys Scr 79 (2009). 035006

15. Attallah, SK, El-Sabbagh, MF, Ali, AT: Isovector fields and similarity solutions of Einstein vacuum equations for rotating fields. Commun Nonl Sci Numer Simul. 12, 1153-1161 (2007). doi:10.1016/j.cnsns.2006.02.004

16. Bluman, GW, Kumei, S: Symmetries and differential equations. In Applied Mathematical sciences, vol. 81,Springer-Verlag, New York (1989)

17. Olver, PJ: Equivalence, Invariance and Symmetry. Cambridge University, Cambridge (1995)

18. Ovsiannikov, LV: Group Analysis of Differential Equations. Cambridge ca-demic Press, New York (1982)

19. Stephani, H, MacCallum, M: Differential equations: Their solutions using symmetries. Cambridge University Press, Cambridge (1989)

20. Yürüsoy, M, Pakdemirli, M: Group classification of a non-Newtonian fluid model using classical approach and equivalence transformations. Int J Nonlinear Mech. 34, 341-346 (1999). doi:10.1016/S0020-7462(98)00037-7

21. Yürüsoy, M, Pakdermirli, M, Noyan, OF: Lie group analysis of creeping flow of a second grade. Int J Nonlinear Mech. 36 , 955-960 (2001). doi:10.1016/S0020-7462(00)00060-3

22. Yürüsoy, M: Similarity solutions for creeping flow and heat transfer in second grade fluids. Int J Nonlinear Mech. 39 665-672 (2004). doi:10.1016/50020-7462(03)00020-9

23. Shahzad, F, Sajid, M, Hayat, T, Ayub, M: Analytic solution for flow of a micropolar fluid. Acta Mech. 188, 93-102 (2007). doi:10.1007/s00707-006-0398-4

24. Mekheimer, KhS, Husseny, SZA, Ali, AT, Abo-Elkhair, RE: Lie Group Analysis and Similarity Solutions for a Couple Stress Fluid with Heat Transfer. J Adv R Appl Math. 2, 1-17 (2010) 
25. Mekheimer, KhS, Husseny, SZA, Ali, AT, Abo-Elkhair, RE: Lie point symmetries and similarity solutions for an electrically conducting Jeffrey fluid. Phys Scr 83 (2011). 015017

26. Mekheimer, KhS, Husseny, SZA, Ali, AT, Abo-Elkhair, RE: Similarity Solution for Flow of a Micro-Polar Fluid Through a Porous Medium. Applications and Applied Mathematics. 6, 2082-2093 (2011)

doi:10.1186/1687-2770-2012-15

Cite this article as: Mekheimer et al:: Lie group analysis and similarity solutions for hydro-magnetic Maxwell fluid through a porous medium. Boundary Value Problems 2012 2012:15.

Submit your manuscript to a SpringerOpen ${ }^{\circ}$ journal and benefit from:

- Convenient online submission

- Rigorous peer review

- Immediate publication on acceptance

- Open access: articles freely available online

- High visibility within the field

- Retaining the copyright to your article

Submit your next manuscript at $\gg$ springeropen.com 